\title{
The hypocholesterolaemic effects of Lactobacillus acidophilus American Type Culture Collection 4356 in rats are mediated by the down-regulation of Niemann-Pick C1-Like 1
}

\author{
Ying Huang ${ }^{1}$, Jinfeng Wang ${ }^{1}$, Yi Cheng ${ }^{2}$ and Yongchen Zheng ${ }^{1 *}$ \\ ${ }^{1}$ Department of Biochemistry and Molecular Biology, The Second Clinical Hospital of Jilin University, Changchun 130041, \\ People's Republic of China \\ ${ }^{2}$ Department of Biochemistry and Molecular Biology, Norman Bethune College of Medicine, Jilin University, Changchun 130021, \\ People's Republic of China \\ (Received 15 December 2009 - Revised 8 March 2010 - Accepted 10 March 2010 - First published online 5 May 2010)
}

\begin{abstract}
The objective of the present study was to evaluate the effect of Lactobacillus acidophilus 4356 on cholesterol metabolism in vivo. Rats were fed a cholesterol-enriched experimental diet with or without L. acidophilus 4356 supplementation at a dose of $10^{9}$ colony-forming units per d. L. acidophilus 4356 feeding significantly lowered total serum cholesterol, LDL-cholesterol and TAG concentrations, but there was no change in the serum HDL-cholesterol concentrations. In addition, total liver cholesterol and TAG were decreased in the L. acidophilus 4356-fed group. The expression of Niemann-Pick C1-Like 1 (NPC1L1) in the duodenum and jejunum was significantly decreased following L. acidophilus 4356 feeding. Lactobacillus acidophilus 4356 increased the population of lactobacilli and bifidobacteria in the small intestine and faeces compared with the control. These results indicate that the probiotic potential of the L. acidophilus 4356 strain in the control of cholesterol is at least partially mediated by the down-regulation of NPC1L1. Furthermore, these results also potentially suggest a new mechanism that is responsible for the cholesterol-reducing effects of probiotics.
\end{abstract}

Lactobacillus acidophilus American Type Culture Collection 4356: Cholesterol-lowering effects: Niemann-Pick C1-Like 1

Elevated concentrations of dietary cholesterol as well as high cholesterol concentrations in the serum are both associated with an increased risk of atherosclerosis and CHD. A reduction as small as $1 \%$ in serum cholesterol concentrations has been shown to decrease the risk of CHD in human subjects by $2-3 \%^{(1)}$. In addition to endogenously synthesised cholesterol, the absorption of dietary cholesterol and the reabsorption of biliary cholesterol in the small intestine also contribute to the regulation of plasma cholesterol concentrations ${ }^{(2)}$. Moreover, reducing the intestinal absorption of dietary and biliary cholesterol can decrease plasma cholesterol concentrations ${ }^{(3)}$.

It has been demonstrated that the molecular mechanism responsible for intestinal cholesterol absorption requires the Niemann-Pick C1-Like 1 (NPC1L1) protein $^{(4)}$. Mice lacking NPC1L1 display a substantial reduction in cholesterol absorption $^{(4)}$, and are completely resistant to both diet-induced hypercholesterolaemia and apoE deficiency-induced atherosclerosis $^{(5,6)}$. In rats, the expression level of NPC1L1 along the length of the small intestine has been correlated with the efficiency of cholesterol absorption, with the highest level being found in the proximal intestine (duodenum and jejunum) and lower expression being found in the distal intestine (ileum) ${ }^{(4)}$.
Lactic acid bacteria such as Lactobacillus acidophilus are important, beneficial micro-organisms in the intestines of healthy human subjects ${ }^{(7)}$, and they have been associated with several probiotic effects in both human subjects and animals $^{(8)}$. In fact, the hypocholesterolaemic effects of probiotics have already been demonstrated in various animal and human trials ${ }^{(9-14)}$. Among the beneficial effects that have been described, the reduction of blood cholesterol concentrations is of particular interest ${ }^{(15)}$. It has been proposed that the mechanisms underlying the hypocholesterolaemic activity of probiotics can be attributed to the inhibition of the absorption of exogenous cholesterol in the small intestine by two mechanisms: through the binding and incorporation of cholesterol by bacterial cells, and through the suppression of bile acid reabsorption mediated by bacterial bile salt hydrolysis ${ }^{(16-19)}$.

Lactobacillus acidophilus is one of the most well-known species of beneficial bacteria that predominantly reside in the small intestine ${ }^{(20)}$. Our previous in vitro study indicated that L. acidophilus 4356 reduces cholesterol absorption by down-regulating the expression of NPC1L1 ${ }^{(21)}$. The present study investigated whether L. acidophilus American Type Culture Collection (ATCC) 4356 affects cholesterol concentrations and NPC1L1 expression in vivo, which may

Abbreviations: ATCC, American Type Culture Collection; LDL-C, LDL-cholesterol; NPC1L1, Niemann-Pick C1-Like 1; TC, total cholesterol

* Corresponding author: Dr Yongchen Zheng, fax +86 431 85636526, email zhengyc@jlu.edu.cn 
provide a possible molecular mechanism for the modulation of cholesterol concentrations.

\section{Materials and methods}

\section{Source and maintenance of bacterial cultures}

Lactobacillus acidophilus strain ATCC 4356 was obtained from ATCC (Rockville, MD, USA). Stock culture was stored in $40 \%(\mathrm{v} / \mathrm{v})$ glycerol at $-80^{\circ} \mathrm{C}$. The organism was subcultured three times before use in sterile de Man, Rogosa, Sharpe broth using $1 \%$ inoculum, and was allowed to grow for $16 \mathrm{~h}$ at $37^{\circ} \mathrm{C}$. The inoculum was stored at $4^{\circ} \mathrm{C}$ between transfers.

Preparation of freeze-dried Lactobacillus acidophilus American Type Culture Collection 4356

Lactobacillus acidophilus ATCC 4356 was grown in de Man, Rogosa, Sharpe broth at $37^{\circ} \mathrm{C}$ for $16 \mathrm{~h}$. The cells were harvested by centrifugation at $2000 \mathrm{~g}$ for $20 \mathrm{~min}$, washed twice with sterile distilled water and frozen at $-80^{\circ} \mathrm{C}$ overnight. The cells were dried under vacuum for $24 \mathrm{~h}$ in a freeze dryer (DC400; Yamato, Tokyo, Japan).

\section{Animal feeding and grouping}

Twenty male Sprague-Dawley rats (4 weeks of age) were obtained from the National Animal Breeding and Research Centre, Beijing, China. The rats were fed a commercial chow (Kangqiao, Inc., Beijing, China), which included $32 \%$ protein, $5 \%$ fat, $2 \%$ fibre, $1.8 \% \mathrm{Ca}, 1.2 \% \mathrm{P}$ and $59 \%$ $\mathrm{N}$-free extract for 1 week. After this adaptation period, rats were divided into two groups consisting of ten rats each. Group A was fed a high-cholesterol diet only, which included $1 \%$ cholesterol (Aoboxing Biotech, Beijing, China), $10 \%$ lard, $5 \%$ sucrose, $0.3 \%$ sodium cholate (Aoboxing Biotech) and $78.5 \%$ chow. Group B was fed a high-cholesterol diet that was identical to that fed to group A supplemented with freeze-dried L. acidophilus ATCC 4356 at a dose of $10^{9}$ colony-forming units per $\mathrm{d}$. Rats were individually housed in metal cages at a controlled temperature $\left(23 \pm 3^{\circ} \mathrm{C}\right)$ and humidity $(55 \pm 10 \%)$ under a $12 \mathrm{~h}$ light-dark cycle. The rats were fed for 4 weeks, during which time, body weight and food intake were recorded daily. After the feeding period, the rats were fasted overnight and used for subsequent testing. Animal experiments were conducted in compliance with the Guide for Care and Use of Laboratory Animals from the National Institutes of Health ${ }^{(22)}$.

\section{Assay for serum lipids}

Blood samples were collected from the tail veins of the rats under diethyl ether anaesthesia on days 0, 7, 14, 21 and 28. Approximately, $1 \mathrm{ml}$ of blood was taken from each rat, transferred to sterile tubes and kept on ice for $30 \mathrm{~min}$. The tubes were then centrifuged at $2000 \mathrm{~g}$ for $20 \mathrm{~min}$ at $4^{\circ} \mathrm{C}$. Collected serum samples were analysed to determine serum total cholesterol (TC), HDL-cholesterol, LDL-cholesterol (LDL-C) and TAG concentrations using a commercial kit (Biosino Biotechnology and Science, Beijing, China).

\section{Assay for liver total cholesterol and TAG}

After 4 weeks on the appropriate diet, all the rats were euthanised. The livers were removed, rinsed with normal saline solution, blot dried with a filter paper and weighed. The liver TC and TAG levels were determined according to the method of Chiu et al. ${ }^{(11)}$.

\section{Preparation of samples for RNA and protein measurements}

After the 4-week feeding period, rats were euthanised with diethyl ether. The small intestines were removed, flushed with ice-cold PBS and cut into three sections of equal length. The sections were slit lengthwise, and the mucosa was gently scraped, frozen in liquid $\mathrm{N}_{2}$ and stored at $-80^{\circ} \mathrm{C}$. Total RNA was extracted from the tissue samples using the RNA STAT-60 (Tel-Test, Friendswood, TX, USA). Total protein was recovered from the organic phase that remained after RNA isolation by precipitating with isopropanol, washing with $0.3 \mathrm{M}$-guanidine hydrochloride in $95 \%$ ethanol and resuspending the protein pellet in $1 \%$ SDS and $50 \mathrm{~mm}$-Tris $-\mathrm{HCl}$, pH $8 \cdot 8^{(23)}$. The RNA concentration was determined by absorbance at $260 \mathrm{~nm}$, and the protein concentration was determined using the Bio-Rad Protein Assay Kit (Hercules, CA, USA).

Measurement of Niemann-Pick C1-Like $1 \mathrm{mRNA}$ in the small intestines by quantitative real-time PCR

Complementary DNA was synthesised using the complementary DNA Synthesis Kit obtained from Invitrogen (Carlsbad, CA, USA) according to the manufacturer's protocol. Semi-quantitative, SYBR Green-based real-time PCR (Applied Biosystems, Foster City, CA, USA) was used to detect transcripts. Forward and reverse primers were mixed in equal amounts, and were used at a final concentration of $0 \cdot 2 \mu \mathrm{M}$. The following primer sequences were used for PCR: rat NPC1L1, forward: $5^{\prime}$-AACAGCGAGAGGCTCACATT-3' and reverse: 5'-AGTGGCGTTCATGCCTGCCT-3'; and rat $\beta$-actin, forward: $5^{\prime}$-ATTGTGATGGACTCCGGAGA- $3^{\prime}$ and reverse: 5'-CAGCTCATAGCTCTTCTCCA-3'. Each experiment was carried out with duplicate samples. The mRNA level in each sample was determined in triplicate. Real-time PCR was performed using the ABI 7500 System for data acquisition, and was analysed using the ABI 7500 System Sequence Detection software. Data are presented as means and standard deviations.

\section{Western blot}

Total protein obtained from the lysates of tissue samples was size fractionated on a $6 \%$ SDS-PAGE ( $40 \mu \mathrm{g} / \mathrm{lane})$, transferred electrophoretically to a nitrocellulose membrane and then analysed by Western blot. Rabbit anti-rat NPC1L1 and anti-rat $\beta$-actin polyclonal antibodies were purchased from Novus Biologicals (Littleton, CO, USA). Immunodetection was carried out with appropriate secondary peroxidase-conjugated antibodies (Pierce Biotechnology, Rockford, IL, USA) and chemiluminescence detection (enhanced chemiluminescence; Amersham, Poole, UK). 
Table 1. Body weight gain, total food intake and food efficiency of rats fed a high-cholesterol diet for 4 weeks

(Mean values and standard deviations)

\begin{tabular}{|c|c|c|c|c|}
\hline & \multicolumn{2}{|c|}{ Group A* } & \multicolumn{2}{|c|}{ Group B† } \\
\hline & Mean & SD & Mean & SD \\
\hline Body weight gain (g) & 124.06 & 3.45 & $122 \cdot 56$ & $5 \cdot 67$ \\
\hline Total food intake (g) & 698.67 & 19.32 & 718.89 & 21.67 \\
\hline Food efficiency (\%)‡ & $17 \cdot 77$ & 0.41 & 17.05 & 0.35 \\
\hline
\end{tabular}

* Group A, high-cholesterol diet only.

† Group B, high-cholesterol diet + Lactobacillus acidophilus American Type Culture Collection 4356.

‡ Food efficiency $(\%)=($ body weight gain/food intake $) \times 100$.

\section{Assay for small intestinal and faecal microflora}

The mucosal samples were scraped from the small intestine for isolation and enumeration of bacteria. Faecal samples for microbial analysis were collected weekly in separate sterile tubes, and were analysed within $1 \mathrm{~h}$ of harvest. Each sample was homogenised using sterile peptone and water diluents. Subsequently, tenfold serial dilutions of each sample were plated in triplicate. Eosin methylene blue agar was used for Escherichia coli, whereas de Man, Rogosa, Sharpe agar was used for lactic acid bacteria, and Bifidobacterium-selective medium agar was used for bifidobacteria. Eosin methylene blue agar and Bifidobacterium-selective medium agar were obtained from Luqiao Tech (Beijing, China).

\section{Statistical analysis}

Data are expressed as the means and standard deviations. The statistical significance of the difference between two means at one particular time point was evaluated using Student's $t$ test. Two-way ANOVA with repeated measures was used to compare the effects over time between the two groups. In these tests, values of $P<0.05$ were considered significant.

\section{Results}

\section{Weight and food intake}

All rats appeared healthy throughout the feeding period. No significant differences in body weight gain, total food intake or food efficiency $(P>0.05)$ were seen between the two groups of rats (Table 1).

\section{Blood lipid analysis}

Table 2 shows the effects of dietary cholesterol and L. acidophilus ATCC 4356 on serum cholesterol and TAG levels in rats. The TC, LDL-C and TAG concentrations observed in group B were significantly decreased than those observed in group A. The HDL-cholesterol concentrations, however, did not show a significant difference between the groups.

\section{Liver lipid analysis}

Table 3 shows the data on weight and lipid content of the liver. Average liver weight was not significantly different between the two groups. However, liver cholesterol and TAG concentrations were significantly lower in group B than in group $\mathrm{A}(P<0 \cdot 05)$.

Lactobacillus acidophilus American Type Culture Collection 4356 inhibits Niemann-Pick C1-Like 1 expression in the small intestine

To address the mechanism underlying $L$. acidophilus 4356-mediated inhibition of cholesterol absorption, mRNA levels of NPC1L1 in the small intestines were assayed (Fig. 1(a)). The level of NPCIL1 mRNA varied in different segments of the rat intestine, with the peak expression being observed in the proximal jejunum. In the duodenal and jejunal segments, NPC1L1 mRNA levels detected in group B were significantly lower than those detected in the control group. These decreases in NPC1L1 levels in group B were also confirmed at the protein level (Fig. 1(b)). In contrast, there was no significant difference in NPCIL1 mRNA levels in the ileal segment.

\section{Microbial populations in the small intestine}

Table 4 shows the counts of total lactobacilli and bifidobacteria in the duodenum, jejunum and ileum in rats fed either the control or the L. acidophilus ATCC 4356-supplemented diet.

Table 2. Serum total cholesterol (TC), LDL-cholesterol (LDL-C) and TAG levels in rats fed a high-cholesterol diet (Mean values and standard deviations)

\begin{tabular}{|c|c|c|c|c|c|c|c|c|c|c|}
\hline & \multicolumn{2}{|c|}{ Day 0} & \multicolumn{2}{|c|}{ Day 7} & \multicolumn{2}{|c|}{ Day 14} & \multicolumn{2}{|c|}{ Day 21} & \multicolumn{2}{|c|}{ Day 28} \\
\hline & Mean & SD & Mean & SD & Mean & SD & Mean & SD & Mean & SD \\
\hline \multicolumn{11}{|l|}{ TC (mg/l) } \\
\hline Group $A^{*}$ & 1824.9 & 63.4 & 1898.5 & $168 \cdot 7$ & $2011 \cdot 5^{\mathrm{a}}$ & $124 \cdot 3$ & $2086 \cdot 3^{\mathrm{a}}$ & 161.5 & $2118 \cdot 7^{\mathrm{a}}$ & 164.9 \\
\hline Group B† & $183 \cdot 87$ & 3.78 & $177 \cdot 45$ & $12 \cdot 98$ & $173.42^{b}$ & $10 \cdot 16$ & $170 \cdot 45^{b}$ & $13 \cdot 21$ & $165 \cdot 97^{\mathrm{b}}$ & $19 \cdot 21$ \\
\hline \multicolumn{11}{|l|}{ LDL-C (mg/l) } \\
\hline Group $A^{*}$ & 951.2 & $52 \cdot 3$ & 983.9 & $122 \cdot 6$ & $1214 \cdot 8^{\mathrm{a}}$ & $116 \cdot 3$ & $1201 \cdot 1^{a}$ & $103 \cdot 2$ & $1238 \cdot 1^{a}$ & $130 \cdot 9$ \\
\hline Group B十 & 934.6 & $36 \cdot 0$ & $916 \cdot 4$ & 84.5 & $921 \cdot 4^{b}$ & $136 \cdot 2$ & $854.9^{b}$ & 94.7 & $798 \cdot 5^{b}$ & 103.6 \\
\hline \multicolumn{11}{|l|}{ TAG (mg/l) } \\
\hline Group A* & 456.5 & $57 \cdot 3$ & $448 \cdot 3$ & $62 \cdot 3$ & $485.2^{\mathrm{a}}$ & 64.5 & $504 \cdot 3^{\mathrm{a}}$ & $73 \cdot 1$ & $519 \cdot 7^{\mathrm{a}}$ & $109 \cdot 7$ \\
\hline Group B $\dagger$ & 418.9 & $36 \cdot 5$ & $429 \cdot 1$ & $47 \cdot 8$ & $321.9^{b}$ & $52 \cdot 1$ & $313 \cdot 1^{b}$ & $45 \cdot 6$ & $342 \cdot 1^{b}$ & $113 \cdot 2$ \\
\hline
\end{tabular}

${ }^{a, b}$ Mean values with unlike superscript letters were significantly different as assessed by ANOVA $(P<0.05)$.

* Group A, high-cholesterol diet only.

† Group B, high-cholesterol diet + Lactobacillus acidophilus American Type Culture Collection 4356. 
Table 3. Hepatic cholesterol and TAG levels in rats fed a highcholesterol diet

(Mean values and standard deviations)

\begin{tabular}{|c|c|c|c|c|}
\hline & \multicolumn{2}{|c|}{ Group A† } & \multicolumn{2}{|c|}{ Group Bł } \\
\hline & Mean & SD & Mean & SD \\
\hline Liver weight (g) & 8.98 & $0 \cdot 12$ & 8.64 & 0.08 \\
\hline Liver cholesterol (mg/g) & $12 \cdot 08$ & 0.06 & $8.32^{*}$ & 0.03 \\
\hline Liver TAG (mg/g) & $33 \cdot 21$ & 0.24 & $27 \cdot 23^{*}$ & 0.14 \\
\hline
\end{tabular}

In both the groups, there was a trend for lactobacilli and bifidobacteria counts to be highest in the ileum than in the more proximal regions of the intestine. In the duodenal, jejunal and ileal segments, both lactobacilli and bifidobacteria counts were significantly greater in rats fed $L$. acidophilus ATCC 4356 than in rats in group A.

\section{Microbial populations in the faeces}

Table 5 shows the effect of $L$. acidophilus ATCC 4356 on the faecal microflora of rats fed a high-cholesterol diet. Significant increases in the total lactobacilli and bifidobacteria populations were observed in the faecal samples of rats fed L. acidophilus ATCC 4356 than in those of the

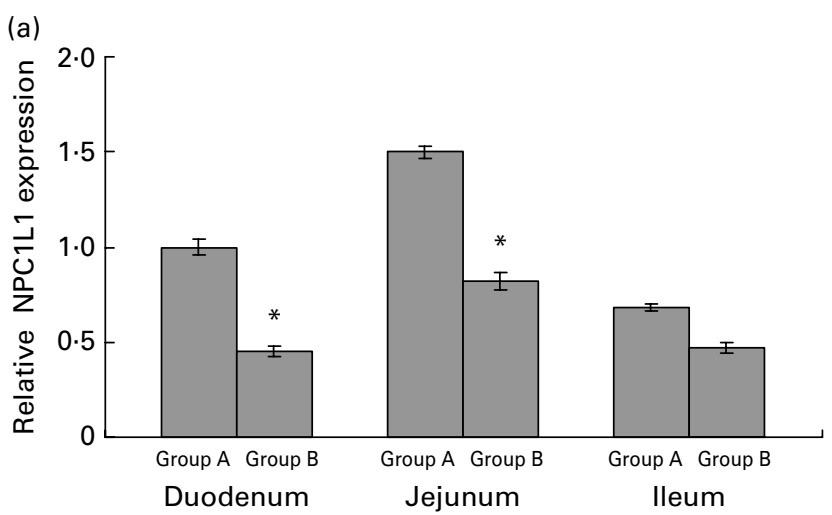

(b)

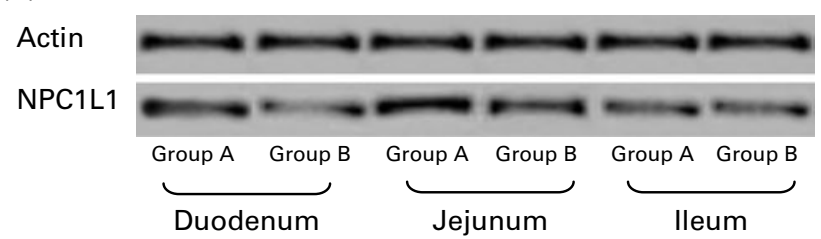

Fig. 1. Consumption of Lactobacillus acidophilus American Type Culture Collection (ATCC) 4356 down-regulates Niemann-Pick C1-Like 1 (NPC1L1) expression in the small intestines of rats. (a) Real-time PCR of NPC1L1 mRNA in the small intestines of rats fed $L$. acidophilus ATCC 4356 (group B) was compared to that in the small intestines of the controls (group A). The data are presented as the means and standard deviations. * Mean values were significantly different compared with the control group by Student's $t$ test $(P<0.05)$. (b) Protein expression of NPC1L1 in the small intestines of rats fed L. acidophilus ATCC 4356 (group B) was compared to that in the small intestines of the control group using Western blot. Actin expression serves as a loading control.
Table 4. Bacterial counts in different regions of the small intestine of rats fed a high-cholesterol diet

(Mean values and standard deviations)

\begin{tabular}{|c|c|c|c|c|}
\hline \multirow[b]{3}{*}{ Organ and bacterial group } & \multicolumn{4}{|c|}{$\begin{array}{c}\text { Bacterial count (log colony-forming } \\
\text { units/g wet weight) }\end{array}$} \\
\hline & \multicolumn{2}{|c|}{ Group A† } & \multicolumn{2}{|c|}{ Group B‡ } \\
\hline & Mean & $\mathrm{SD}$ & Mean & SD \\
\hline \multicolumn{5}{|l|}{ Duodenum } \\
\hline Lactobacilli & 3.91 & 0.17 & $5 \cdot 68^{*}$ & 0.23 \\
\hline Bifidobacteria & 2.65 & 0.21 & $3.93^{*}$ & 0.13 \\
\hline \multicolumn{5}{|l|}{ Jejunum } \\
\hline Lactobacilli & 4.39 & 0.31 & $5 \cdot 87^{\star}$ & 0.26 \\
\hline Bifidobacteria & 2.94 & 0.27 & $4 \cdot 16^{*}$ & 0.31 \\
\hline \multicolumn{5}{|l|}{ Ileum } \\
\hline Lactobacilli & 4.84 & 0.34 & $6 \cdot 51^{*}$ & 0.38 \\
\hline Bifidobacteria & 3.41 & 0.23 & $4.65^{\star}$ & 0.33 \\
\hline
\end{tabular}

* Mean values were significantly different when compared with the control group by Student's $t$ test $(P<0.05)$.

$\dagger$ Group A, high-cholesterol diet only.

$\ddagger$ Group B, high-cholesterol diet + Lactobacillus acidophilus American Type Culture Collection 4356.

control group. However, faecal levels of lactobacilli and bifidobacteria in rats fed a cholesterol-enriched diet without L. acidophilus 4356 supplementation remained unchanged throughout the experimental period. The total E. coli count did not significantly change between the two groups during the entire experimental period.

\section{Discussion}

High concentrations of TC and LDL-C are strongly associated with an increased risk of CHD. A reduction in the TC and LDL-C concentrations in hypercholesterolaemic men has been shown to reduce the incidence of $\mathrm{CVD}^{(24)}$. The modification of diet, such as the inclusion of fermented dairy products or lactic acid bacteria-containing dairy products, is one way through which serum cholesterol can be reduced ${ }^{(12)}$. In agreement with previous reports ${ }^{(25-27)}$, the present study demonstrated that ingestion of L. acidophilus ATCC 4356 results in a reduction of the serum TC, LDL-C and TAG concentrations of rats fed a high-cholesterol diet. Since significant reductions in cholesterol and TAG concentrations were also observed in the liver of rats in the L. acidophilus ATCC 4356-fed group, it can be suggested that the cholesterol concentration was actually reduced, and not merely re-distributed between the blood and liver. No significant differences in HDL-cholesterol concentrations were observed in our study in agreement with previous results in rats and human subjects reported by Abd El-Gawad et al. ${ }^{(28)}$, Fukushima \& Nakano ${ }^{(29)}$ and Wang et al. ${ }^{(13)}$.

In contrast to the present results, some researchers ${ }^{(30,31)}$ have not observed hypocholesterolaemic effects from lactic acid bacteria consumed by mice or human subjects. Many researchers ${ }^{(32,33)}$ have suggested that these conflicting results may be due to the different properties of the cultures used (e.g. acid and bile tolerance). Other important factors that may affect the results include the amount of bacteria ingested, the cholesterol content of the diet under study, the animal used and the length of the feeding period. 
Table 5. Population of lactobacilli, bifidobacteria, and Escherichia coli from faeces of rats fed a high-cholesterol diet (Mean values and standard deviations)

\begin{tabular}{|c|c|c|c|c|c|c|c|c|c|c|}
\hline \multirow{2}{*}{$\begin{array}{l}\text { Bacterial count (log colony-forming units } / g \\
\text { wet faecal samples) }\end{array}$} & \multicolumn{2}{|c|}{ Day 0} & \multicolumn{2}{|c|}{ Day 7} & \multicolumn{2}{|c|}{ Day 14} & \multicolumn{2}{|c|}{ Day 21} & \multicolumn{2}{|c|}{ Day 28} \\
\hline & Mean & SD & Mean & SD & Mean & SD & Mean & SD & Mean & SD \\
\hline \multicolumn{11}{|l|}{ Lactobacilli } \\
\hline Group A* & 8.52 & 0.04 & 8.62 & 0.07 & $8 \cdot 51^{\mathrm{b}}$ & 0.03 & $8 \cdot 31^{b}$ & 0.02 & $8 \cdot 54^{\mathrm{b}}$ & 0.05 \\
\hline Group B† & 8.87 & 0.03 & 8.89 & 0.04 & $9.89^{a}$ & 0.03 & $10 \cdot 03^{a}$ & 0.02 & $10 \cdot 06^{\mathrm{a}}$ & 0.03 \\
\hline \multicolumn{11}{|l|}{ Bifidobacteria } \\
\hline Group $A^{*}$ & 7.83 & 0.01 & 7.63 & 0.08 & $7 \cdot 55^{\mathrm{b}}$ & 0.03 & $7 \cdot 68^{b}$ & 0.04 & $7 \cdot 82^{b}$ & 0.05 \\
\hline Group B† & 7.98 & 0.06 & 8.21 & 0.03 & $8 \cdot 87^{a}$ & 0.08 & $9 \cdot 16^{\mathrm{a}}$ & 0.06 & $9 \cdot 11^{\mathrm{a}}$ & 0.06 \\
\hline
\end{tabular}

${ }^{a, b}$ Mean values with unlike superscript letters were statistically different as assessed by ANOVA $(P<0.05)$.

* Group A, high-cholesterol diet only.

† Group B, high-cholesterol diet + Lactobacillus acidophilus American Type Culture Collection 4356.

Since cholesterol absorption primarily occurs in the duodenum and proximal jejunum, with little absorption by the ileal segment of the intestine ${ }^{(34,35)}$, we investigated NPC1L1 mRNA expression along the duodenum-ileum axis. The level of NPC1L1 mRNA varied in the different segments of rat intestine, with the peak expression being detected in the proximal jejunum, a result that is in agreement with a study done by Altmann et al. ${ }^{(4)}$. Lactobacillus and Bifidobacterium counts in the small intestines of rats fed L. acidophilus ATCC 4356 were significantly greater than those in the small intestines of rats solely fed the high-cholesterol diet. Moreover, NPC1L1 mRNA levels in the duodenal and jejunal segments of rats in the L. acidophilus ATCC 4356-fed group were significantly lower than those in the duodenal and jejunal segments of the control group. In contrast to previous studies, the present results indicate that $L$. acidophilus ATCC 4356 is able to reduce cholesterol absorption by inhibiting NPC1L1 mRNA transcription in the small intestine.

In our study, the number of faecal lactobacilli and bifidobacteria in the L. acidophilus ATCC 4356-fed group is greater than that observed in the high-cholesterol group. This indicates that $L$. acidophilus ATCC 4356 can successfully tolerate the gastric acid and bile salts of the small intestines, and still retain biological activity. As Donnet-Hughes et al. ${ }^{(36)}$ have suggested, bacterial survival in the faeces following oral administration reflects successful bowel colonisation and proliferation that are required for the initiation of biological effects.

Many studies have shown that lactic acid bacteria inhibit the proliferation of pathogenic bacteria, improve the intestinal microflora composition, reduce the risk of diseases and promote the health of the host ${ }^{(8,37,38)}$. However, in rats of both group $\mathrm{A}$ and group $\mathrm{B}$, the faecal $E$. coli level was constant throughout the feeding period. These results indicate that the growth of E. coli is not inhibited by L. acidophilus ATCC 4356 in vivo.

In conclusion, in addition to contributing to a healthy microbial balance in the bowels, L. acidophilus ATCC 4356 also exerted a significant hypocholesterolaemic effect on rats fed a high-cholesterol diet through inhibition of NPC1L1 expression in the small intestines. These findings suggest a novel mechanism that may underlie probiotic-mediated cholesterol reduction. The mechanism proposed here differs from conclusions drawn by previous studies, which have implicated the incorporation of cholesterol into cellular membranes or the deconjugation of bile salts during bacterial growth as the underlying mechanism. However, it will be necessary to conduct more extensive animal studies, using varying doses of bacteria over longer times, to fully assess the long-term cholesterol-lowering potential of L. acidophilus ATCC 4356.

\section{Acknowledgements}

The authors gratefully acknowledge financial support from the Scientific Foundation of Jilin Province (grant no. 2008026). The authors performed this experiment together, and contributed to the completion of the manuscript. Y. H. designed the study, performed the data analysis and contributed to the drafting of the paper. Y. Z., J. W. and Y. C. performed the data analysis. The authors declare no conflict of interest.

\section{References}

1. Manson JE, Tosteson H, Ridker PM, et al. (1992) The primary prevention of myocardial infarction. $N$ Engl $J$ Med 326, 1406-1416.

2. Wilson MD \& Rudel LL (1994) Review of cholesterol absorption with emphasis on dietary and biliary cholesterol. J Lipid Res 35, 943-955.

3. Gylling H \& Miettinen TA (1995) The effect of cholesterol absorption inhibition on low density lipoprotein cholesterol level. Atherosclerosis 117, 305-308.

4. Altmann SW, Davis HR Jr, Zhu LJ, et al. (2004) Niemann-Pick C1 Like 1 protein is critical for intestinal cholesterol absorption. Science 303, 1201-1204.

5. Davis HR Jr, Hoos LM, Tetzloff G, et al. (2007) Deficiency of Niemann-Pick C1 Like 1 prevents atherosclerosis in ApoE - /- mice. Arterioscler Thromb Vasc Biol 27, 841-849.

6. Davis HR Jr, Zhu LJ, Hoos LM, et al. (2004) Niemann-Pick C1 Like 1 (NPC1L1) is the intestinal phytosterol and cholesterol transporter and a key modulator of whole-body cholesterol homeostasis. J Biol Chem 279, 33586-33592.

7. Macfarlane GT \& Cummings JH (2002) Probiotics, infection and immunity. Curr Opin Infect Dis 15, 501-506.

8. Fuller R (1989) Probiotics in man and animals. J Appl Bacteriol 66, 365-378.

9. Simons LA, Amansec SG \& Conway P (2006) Effects of Lactobacillus fermentum on serum lipids in subjects with elevated serum cholesterol. Nutr Metab Cardiovasc Dis 16, $531-535$ 
10. Pereira DI \& Gibson GR (2002) Effects of consumption of probiotics and prebiotics on serum lipid levels in humans Crit Rev Biochem Mol Biol 37, 259-281.

11. Chiu CH, Lu TY, Tseng YY, et al. (2006) The effects of Lactobacillus-fermented milk on lipid metabolism in hamsters fed on high-cholesterol diet. Appl Microbiol Biotechnol 71, 238-245.

12. Park YH, Kim JG, Shin YW, et al. (2007) Effect of dietary inclusion of Lactobacillus acidophilus ATCC 43121 on cholesterol metabolism in rats. J Microbiol Biotechnol 17, 655-662.

13. Wang Y, Xu N, Xi A, et al. (2009) Effects of Lactobacillus plantarum MA2 isolated from Tibet kefir on lipid metabolism and intestinal microflora of rats fed on high-cholesterol diet. Appl Microbiol Biotechnol 84, 341-347.

14. Xiao JZ, Kondo S, Takahashi N, et al. (2003) Effects of milk products fermented by Bifidobacterium longum on blood lipids in rats and healthy adult male volunteers. J Dairy Sci 86, 2452-2461.

15. Ebringer L, Ferencík M \& Krajcovic J (2008) Beneficial health effects of milk and fermented dairy products - review. Folia Microbiol (Praha) 53, 378-394.

16. Liong MT \& Shah NP (2005) Bile salt deconjugation ability, bile salt hydrolase activity and cholesterol co-precipitation ability of lactobacilli strains. J Dairy Sci 15, 391-398.

17. Corzo G \& Gilliland SE (1999) Bile salt hydrolase activity of three strains of Lactobacillus acidophilus. J Dairy Sci $\mathbf{8 2}$ $472-480$.

18. Noh DO, Kim SH \& Gilliland SE (1997) Incorporation of cholesterol into the cellular membrane of Lactobacillus acidophilus ATCC 43121. J Dairy Sci 80, 3107-3113.

19. Buck LM \& Gilliland SE (1994) Comparisons of freshly isolated strains of Lactobacillus acidophilus of human intestinal origin for ability to assimilate cholesterol during growth. J Dairy Sci 77, 2925-2933.

20. Robins-Brown RM \& Levine M (1981) The fate of ingested lactobacilli in the proximal small intestine. Am J Clin Nutr 34, 514-519.

21. Huang Y \& Zheng Y (2010) The probiotic Lactobacillus acidophilus reduces cholesterol absorption through the downregulation of Niemann-Pick C1 Like 1 in Caco-2 cells. $\mathrm{Br} J$ Nutr 103, 473-478.

22. National Institutes of Health (1996) Guide for the Care and Use of Laboratory Animals. Washington, DC: National Academy Press.

23. Banerjee S, Smallwood A \& Chambers AE, et al. (2003) Quantitative recovery of immunoreactive proteins from clinical samples following RNA and DNA isolation. Biotechniques 35, $450-452,454,456$.
24. Probstfield JL \& Rifkind BM (1991) The lipid research clinics coronary primary prevention trial: design, results, and implications. Eur J Clin Pharmacol 40, Suppl. 1, S69-S75.

25. Danielson AD, Peo ER Jr, Shahani KM, et al. (1989) Anticholesteremic property of Lactobacillus acidophilus yogurt fed to mature boars. J Anim Sci 67, 966-974.

26. Gilliland SE, Nelson CR \& Maxwell C (1985) Assimilation of cholesterol by Lactobacillus acidophilus. Appl Environ Microbiol 49, 377-381.

27. Usman \& Hosono A (2000) Effect of administration of Lactobacillus gasseri on serum lipids and fecal steroids in hypercholesterolemic rats. J Dairy Sci 83, 1705-1711.

28. Abd El-Gawad IA, El-Sayed EM, Hafez SA, et al. (2005) The hypocholesterolaemic effect of milk yoghurt and soy-yoghurt containing bifidobacteria in rats fed on a cholesterol-enriched diet. Int Dairy J 15, 37-44.

29. Fukushima M \& Nakano M (1996) Effects of a mixture of organisms, Lactobacillus acidophilus or Streptococcus faecalis on cholesterol metabolism in rats fed on a fat- and cholesterol-enriched diet. Br J Nutr 76, 857-867.

30. Thompson LU, Jenkins DJ, Amer MA, et al. (1982) The effect of fermented and unfermented milks on serum cholesterol. Am J Clin Nutr 36, 1106-1111.

31. St-Onge MP, Farnworth ER, Savard T, et al. (2002) Kefir consumption does not alter plasma lipid levels or cholesterol fractional synthesis rates relative to milk in hyperlipidemic men: a randomized controlled trial. BMC Complement Altern Med 2, 1-7.

32. Akalin AS, Gönç S \& Düzel S (1997) Influence of yogurt and acidophilus yogurt on serum cholesterol levels in mice. J Dairy Sci 80, 2721-2725.

33. Taranto MP, Medici M, Perdigon G, et al. (1998) Evidence for hypocholesterolemic effect of Lactobacillus reuteri in hypercholesterolemic mice. J Dairy Sci 81, 2336-2340.

34. Grundy SM (1983) Absorption and metabolism of dietary cholesterol. Апnи Rev Nutr 3, 71-96.

35. Borgström B (1960) Studies on intestinal cholesterol absorption in the human. $J$ Clin Invest 39, 809-815.

36. Donnet-Hughes A, Rochat F, Serrant P, et al. (1999) Modulation of nonspecific mechanisms of defense by lactic acid bacteria: effective dose. J Dairy Sci 82, 863-869.

37. Seow SW, Cai S, Rahmat JN, et al. (2010) Lactobacillus rhamnosus GG induces tumor regression in mice bearing orthotopic bladder tumors. Cancer Sci 101, 751-758.

38. van Winsen RL, Keuzenkamp D, Urlings BA, et al. (2002) Effect of fermented feed on shedding of Enterobacteriaceae by fattening pigs. Vet Microbiol 87, 267-276. 